Check for updates

Cite this: Phys. Chem. Chem. Phys., 2018, 20, 9616

Received 20th February 2018, Accepted 16th March 2018

DOI: $10.1039 / c 8 c p 01147 h$

rsc.li/pccp

\title{
Tuning the surface of Au nanoparticles using poly(ethylene glycol)-block-poly(propylene glycol)-block-poly(ethylene glycol): enzyme free and label free sugar sensing in serum samples using resonance Rayleigh scattering spectroscopy
}

\begin{abstract}
Riham El Kurdi and Digambara Patra (D) *
Poly(ethylene glycol)-block-poly(propylene glycol)-block-poly(ethylene glycol) (F-108) functionalized gold nanoparticles (Au NPs) have been successfully synthesized. During synthesis it is found that an increase in the F-108 concentration contributes to agglomeration in the media, increasing the size of the $\mathrm{Au}$ particles, and boosting the curcumin concentration leads to a higher density of functional groups, resulting in smaller Au NPs. FT-IR analysis reveals that the hydroxyl and phenolic groups of curcumin and F-108 are involved during the functionalization of Au surfaces. Enhancement in the fluorescence/RRS intensity is due to the combination of the influence of the shape/size of the Au NPs as well as the extent of curcumin conjugation at the interface of the Au NP surface and F-108. The presence of sugar molecules remarkably boosts the RRS intensity without significantly affecting the fluorescence and surface plasmon absorbance of the Au NPs; in contrast, the RRS intensity of standard CTAB functionalized Au NPs is unaffected by glucose molecules indicating that the functionalization of F-108 at Au surfaces is crucial. Interestingly, no interference from other potential interferents and antioxidant substances like ascorbic acid, creatinine and acetaminophen is observed. This method is simple and fast, and offers a wider linear dynamic range, 0-10 $\mathrm{mM}$, that is applicable under physiological conditions and in serum samples. It is stable and provides an excellent recovery for serum samples, thus, potentially it can be useful in this field due to its low energy consumption, enzyme free assay, fast response time, better selectivity and sensitivity.
\end{abstract}

\section{Introduction}

Nanosized noble metal particles, due to their high surface to bulk ratio and quantum-size effects, ${ }^{1}$ present many novel properties, such as high catalytic activities and/or interesting optical properties. $^{2}$ Gold nanoparticles are of great importance in scientific research and industrial applications. ${ }^{3}$ Colloidal gold sols were first formally studied by Faraday in $1857 .{ }^{4}$ Colloidal and bulk gold exhibit several differences. Most notable are the surface charges and high surface areas of the colloidal gold sols. ${ }^{1}$ Gold nanoparticles are colloidal suspensions of gold particles of nanometer sizes. ${ }^{5}$ Therefore, the potential of gold nanoparticles relies on accurate control of the particle size, particle distribution, and stability. ${ }^{6}$ Accordingly, considerable effort has been focused on the development of synthetic techniques for adjusting the metal nanoparticle size, distribution and

Department of Chemistry, American University of Beirut, Beirut, Lebanon. E-mail: dp03@aub.edu.lb; Fax:+9611365217; Tel: +9611350000 ext. 3985 shape. ${ }^{7}$ The size of gold nanoparticles (Au NPs) depends on the rate of gold(III) reduction, suggesting that this rate affects the initial nucleation of gold particles. ${ }^{8}$ However, the rates of gold(III) reduction are influenced by the cavitation phenomenon, and hence, are dependent on reaction parameters. The size of the gold particles is parallel to the initial rate of gold(III) reduction, where the higher the rate of reduction, the smaller the particles. ${ }^{9}$ Thus, the distribution of the Au NPs depends strongly on the size of the particles. ${ }^{10}$

As per the shape, a variety of anisotropic gold nanoparticle shapes, such as, spheres, rods, cubes, prisms, and branched nanoparticles can be synthesized. ${ }^{11}$ The formation of these various shapes is likely the outcome of the interplay between the faceting tendency of the stabilizing agent and the growth kinetics, which means the rate of supply of $\mathrm{Au}^{0}$ to the crystallographic planes. ${ }^{12}$ As shown in the literature, many studies focus on the development of different routes to synthesize gold nanoparticles. ${ }^{8,9,13,14}$ It was found that the efficacy of the reduction of $\mathrm{HAuCl}_{4}$ in the presence of surfactants such as 
sodium dodecyl sulfate, amines ${ }^{15}$ and chitosan, ${ }^{16}$ is related to the concentration of the surfactant in solution. ${ }^{8}$ Hence, the use of stabilizing agents also confines the growth in the nanometer regime and prevents agglomeration. ${ }^{9}$ Among the common stabilizing agents, trisodium citrate dihydrate is used as both a reducing agent and stabilizer of the gold nanoparticles, where citrate ions bind physically at gold surfaces and stabilize the particles in the suspension. Sodium dibasic tartrate, hexadecyltrimethyl ammonium bromide (CTAB) and sodium borohydride can be used as a reducing agent for gold precursors and as a stabilizer for Au NPs in a milder way. ${ }^{9}$ However, these chemicals can be harmful to the environment, producing a toxic reagent with purification difficulties. New green chemistry strategies to produce Au NPs without using toxic chemicals are being actively considered for their biomedical applications. ${ }^{17,18}$ Curcumin, derived from the rhizome of turmeric, is nontoxic and safe. Recently the focus on curcumin has been exponentially growing due to its potential use in medicine and as supplements. ${ }^{7,19-24}$ However, our group has been exploiting curcumin as a green synthetic route to prepare and tune the optical, catalytic and adsorption properties of metal and metal oxide nanoparticles. ${ }^{25-30}$ For example, we have successfully shown the reduction and stabilization of gold nanoparticles using curcumin. However, reducing the $\mathrm{Au}(\mathrm{III})$ ion using curcumin alone is not sufficient for wider applicability and functionalization of the surface of $\mathrm{Au}$ nanoparticles may be necessary.

Polymers are macromolecules composed of a large number of repeating units organized in a chain-like molecular architecture exhibiting a variety of compositions, structures, and properties. $^{31}$ In fact, polymers are being used in nanoparticle systems to generate nanoparticles suited for each specific biomedical application. This is due to the multiplicity of compositions, structures and properties. Hence, the main use of polymeric nanoparticles is in biosensing assays, although they are also used in drug delivery and bioimaging. ${ }^{32}$ Furthermore, natural polymers and synthetic polymers such as $\mathrm{N}$-(2-hydroxypropyl)-methacrylamide, copolymer, poly(ethylene glycol), poly(lactic acid-glycolic acid), and poly(lactic acid) are used for nanoparticle preparation or/and stabilization. ${ }^{31,33}$ Generally, there are four strategies ${ }^{34}$ widely used for creating polymer functionalized nanoparticles including a "grafting from" approach using polymer chain growth from small initiators attached to Au NPs, ${ }^{35}$ "Grafting To", which enables the onepot synthesis of $\mathrm{Au}$ NPs stabilized by sulfur containing polymers, "36 "physisorption" using block copolymer micelles as water-soluble polymers or star block copolymers ${ }^{37}$ and finally "post-modification of pre-formed Au NPs", 38

Biosensing applications cover a very broad range of biologically relevant materials including bioanalytes found in living organisms, such as, cholesterol, uric acid, DNAs, RNAs, cells, proteins, organelles and glucose. ${ }^{39-42}$ However, glucose, at its very core, is a carbohydrate. It is also the most important sugar in human metabolism, the reaction and breakdown of chemicals to maintain our living state. Within the brain itself, it is also the primary source of energy (along with glycogen) used to function. ${ }^{43}$ The normal blood glucose level (tested while fasting) for non-diabetics should be between 3.9 and $5.5 \mathrm{mM}$. The mean normal blood glucose level in humans is about $5.5 \mathrm{mM}$. Blood sugar levels for those without diabetes and who are not fasting should be below $6.9 \mathrm{mM}$. The blood glucose target range for diabetics, according to the American Diabetes Association, should be 5.0-7.2 mM before meals, and less than $10 \mathrm{mM}$ after meals. ${ }^{44}$ Hence, the need for ultrasensitive bioassays and the trend towards miniaturized assays of glucose has made the biofunctionalization of nanomaterials become one of the hottest fields. ${ }^{45}$ Within the group of noble metal nanoparticles, gold nanoparticles are mostly used for biosensor applications due to their biocompatibility, their optical and electronic properties, and their relatively simple production and modification. ${ }^{46}$ Moreover, the use of enzymes during biosensing requires special handling, training and care, which has led to demand for an enzyme free stable sensing scheme. A surface plasmon resonance sensor based on gold nanoparticles has been recently used for the detection of proteins, antibodies and nucleic acids. The several field-enhancements associated with surface plasmon resonance are also explored in many surface analytical techniques such as surface enhanced Raman spectroscopy, surface enhanced infrared absorption spectroscopy, surface-enhanced fluorescence and surface plasmonenhanced diffraction. ${ }^{3}$ The aim of this work is to prepare poly(ethylene glycol)-block-poly(propylene glycol)-block-poly(ethylene glycol) (F-108) stabilized Au NPs for the determination of sugar. Thus, in this work, we explore the functionalization of gold nanoparticles using F-108 through a one pot green synthetic procedure using curcumin as the reducing agent. The optimum concentration of these reagents during the preparation of Au NPs is established. The reaction conditions have been optimized. It has been shown that unlike CTAB functionalized Au NPs prepared using a similar procedure, F-108 functionalized Au NPs successfully estimate sugar in normal and serum samples in the physiological concentration range using an enzyme free environment. This method is simple, stable, and selective and offers the excellent linear dynamic range required under physiological conditions.

\section{Materials and methods}

\subsection{Materials}

Gold(III) chloride hydrate (99.99\% trace metal basis), F-108, curcumin, silver nitrate and galactose were purchased from Sigma Aldrich. Acetaminophen and fructose were from Acros and glucose was from Riedel-de Haën. Finally, creatinine was obtained from Merck. However, all the chemicals were used without any further purification and dissolved in double distilled water; except for curcumin, which was insoluble in water, and was dissolved in methanol.

\subsection{Synthesis of gold nanoparticles}

The preparation of gold nanoparticles was performed as described below using different concentrations of curcumin and polymer (F-108) with or without silver nitrate in neutral media. Briefly, $50 \mu \mathrm{M}$ of $\mathrm{F}-108$ was dissolved in $15 \mathrm{~mL}$ of double 
distilled water heated at $60{ }^{\circ} \mathrm{C}$. The solution was cooled down to $45{ }^{\circ} \mathrm{C}$ and kept for 2 minutes in water. After this $1 \mathrm{~mL}$ of $4 \mathrm{mM}$ $\mathrm{AgNO}_{3}$ prepared separately was added and the solution was kept for 15 minutes in water at $45{ }^{\circ} \mathrm{C}$. To this, $1 \mathrm{mM}$ of $\mathrm{HAuCl}_{4}$ prepared separately in $15 \mathrm{~mL}$ of water was added and the mixture was stirred for $2 \mathrm{~min}$ at $400 \mathrm{rpm}$. Finally, $10 \mathrm{mM}$ of curcumin dissolved in methanol was added to the solution. The final solution was kept for 1 day at $45{ }^{\circ} \mathrm{C}$. To end our procedure, the solution was centrifuged first at $168 \times g$ for 5 min to get rid of unreacted curcumin and subsequently at $37800 \times g$ for $25 \mathrm{~min}$ to precipitate the gold.

\subsection{Characterization and spectroscopic analysis}

The absorption spectra were recorded at room temperature using a JASCO V-570 UV-VIS-NIR spectrophotometer in the wavelength range of $200-600 \mathrm{~nm}$ in a $3 \mathrm{~mL}$ cuvette. The X-ray diffraction (XRD) data were collected using a Bruker $\mathrm{d} 8$ discover $\mathrm{X}$-ray diffractometer equipped with $\mathrm{Cu}-\mathrm{K} \alpha$ radiation $(\lambda=1.5405 \AA)$. The monochromator used was a Johansson type monochromator. Scanning electron microscopy (SEM) analysis was performed using a Tescan, Vega 3 LMU with an Oxford EDX detector (Inca XmaW20). The accelerating voltage was $5 \mathrm{kV}$ with a magnification of $500 \mathrm{~nm}$. In short, a few drops of gold solution were deposited on an aluminum stub and coated with carbon conductive adhesive tape. FT-IR spectra were recorded on a FT-IR-Raman spectrometer. A Thermo Nicolet 4700 Fourier Transform Infrared spectrometer equipped with a Class 1 laser was used for this purpose. The $\mathrm{KBr}$ pellet technique was applied to perform the transmission experiments in the range between 3200 and $700 \mathrm{~cm}^{-1}$. Thermogravimetric Analysis (TGA) was performed using a Netzsch TGA 209 in the temperature range of 30 to $800{ }^{\circ} \mathrm{C}$ with an increase of $10{ }^{\circ} \mathrm{C} \mathrm{min}{ }^{-1}$ under a $\mathrm{N}_{2}$ atmosphere. A synchronous fluorescence scan was performed using a Jobin-Yvon-Horiba Fluorolog III fluorometer and the FluorEssence program. The excitation source was a $100 \mathrm{~W}$ Xenon lamp, and the detector used was an R-928 instrument operating at a voltage of $950 \mathrm{~V}$ by keeping the excitation and emission slits width at $5 \mathrm{~nm}$. The wavelength interval was kept at $0 \mathrm{~nm}$ $(\Delta \lambda=0 \mathrm{~nm})$ to measure the resonance Rayleigh scattering spectrum. The collected Au nanoparticles were mixed with $10 \mathrm{~mL}$ of water and stored. From this solution $200 \mu \mathrm{L}$ was transferred and diluted to $3 \mathrm{~mL}$ and the measurement was carried out in a $3 \mathrm{~mL}$ cuvette.

\subsection{Sample for glucose determination}

Stock solutions of $1 \mathrm{mM}$ and $25 \mathrm{mM}$ of glucose were prepared in order to make up several solutions with a known concentration in the range 0 to $10 \mathrm{mM}$. Real samples were tested by spiking $30 \mu \mathrm{L}$ of the sample with different concentrations of glucose from 0 to $10 \mathrm{mM}$. To investigate the interference, several concentrations of ascorbic acid, acetaminophen, creatinine, fructose and galactose were measured. All the experiments were performed by adding a complementary quantity of water to keep the concentration of Au NPs constant in a total volume of $3 \mathrm{~mL}$.

\section{Results and discussion}

\subsection{One pot synthesis of Au NPs}

To optimize the synthesis of metal nanoparticles, it is necessary to take advantage of reducing agents that can be efficient as a stabilizer of nanoparticles to avoid an excess of reagents and product purity difficulties. Polymers emerged as interesting alternatives playing the role of stabilizing or capping agents preventing the flocculation or coagulation of the formed nanoparticles providing a higher energetic barrier against aggregation. The green synthesis of gold nanoparticles was performed in double distilled water in neutral media (the $\mathrm{pH}$ was adjusted to 7 after adding $\mathrm{HAuCl}_{4}$ ) at $45{ }^{\circ} \mathrm{C}$ as illustrated in Scheme 1.

The synthesis has been carried out by reacting curcumin with $\mathrm{HAuCl}_{4}$. The polymeric micelles formed by F-108 were helpful to dissolve the hydrophobic curcumin. Once the Au salt was reduced by curcumin in F-108 micellar media, Au NPs were produced. According to Sindhu et al., ${ }^{47}$ the formation of curcumin functionalized gold nanoparticles includes six steps starting with the deprotonation, reduction, nucleation, growth, and cleavage, and ending with the maturation of the nanoparticles. After the dissociation of a hydrogen ion from enolic curcumin hydroxyl groups, $\mathrm{Cur}^{-3}$ is formed. Then gold is reduced from $\mathrm{Au}^{3+}$ to $\mathrm{Au}^{0}$ by the electrons on the $\mathrm{O}^{-}$ion in the curcumin molecule. The $\mathrm{Au}^{0}$ atoms form clusters which cleave immediately into smaller fragments due to their instability. Finally, spherical and solid nanoparticles are formed. Hence, the F-108 polymer was used as a capping agent, since it can have a stabilizing effect on the Au NP synthesis.

Localized Surface Plasmon Resonance (SPR) absorption for Au NPs appears at a wavelength $>520 \mathrm{~nm}$, depending on the shape and size of the Au nanoparticles, which is due to the fact that at the localized SPR wavelength gold nanoparticles exhibit an enormous electric field enhancement extended from their surface. ${ }^{3,48}$ During synthesis, the polymer and curcumin concentrations had a primary role in controlling the shape and the size of the particles formed. Therefore, different concentrations were tested for the polymer and curcumin in the range of $0-1 \mathrm{mM}$ and $0-50 \mathrm{mM}$, respectively. In fact, F-108 in the absence of curcumin did not induce the formation of gold nanoparticles, and it was also further confirmed that no precipitate of $\mathrm{Au}$ NPs was obtained after centrifugation, and no colour change compared to the initial reaction system was observed. However, at a high concentration of polymer (above $1 \mathrm{mM}$ ) the micelles formed an insoluble gel. This situation is almost similar to the high concentration of curcumin, $>50 \mathrm{mM}$, when curcumin precipitated in the media.

\subsection{Effect of F-108 concentration}

As per the effect of F-108 concentration during the synthesis of $\mathrm{Au}$ NPs while keeping the curcumin concentration constant at $10 \mathrm{mM}$, the localized SPR spectrum showed a red shift in the localized SPR band of the formed Au particles and a decrease in the absorbance values as the concertation of the polymer increased. At $1 \mathrm{mM}$ of F-108, a clear peak was obtained at $\lambda_{\max }^{\mathrm{SPR}}=544 \mathrm{~nm}$ which shifted to $\lambda_{\max }^{\mathrm{SPR}}=537 \mathrm{~nm}$ on decreasing the 
A
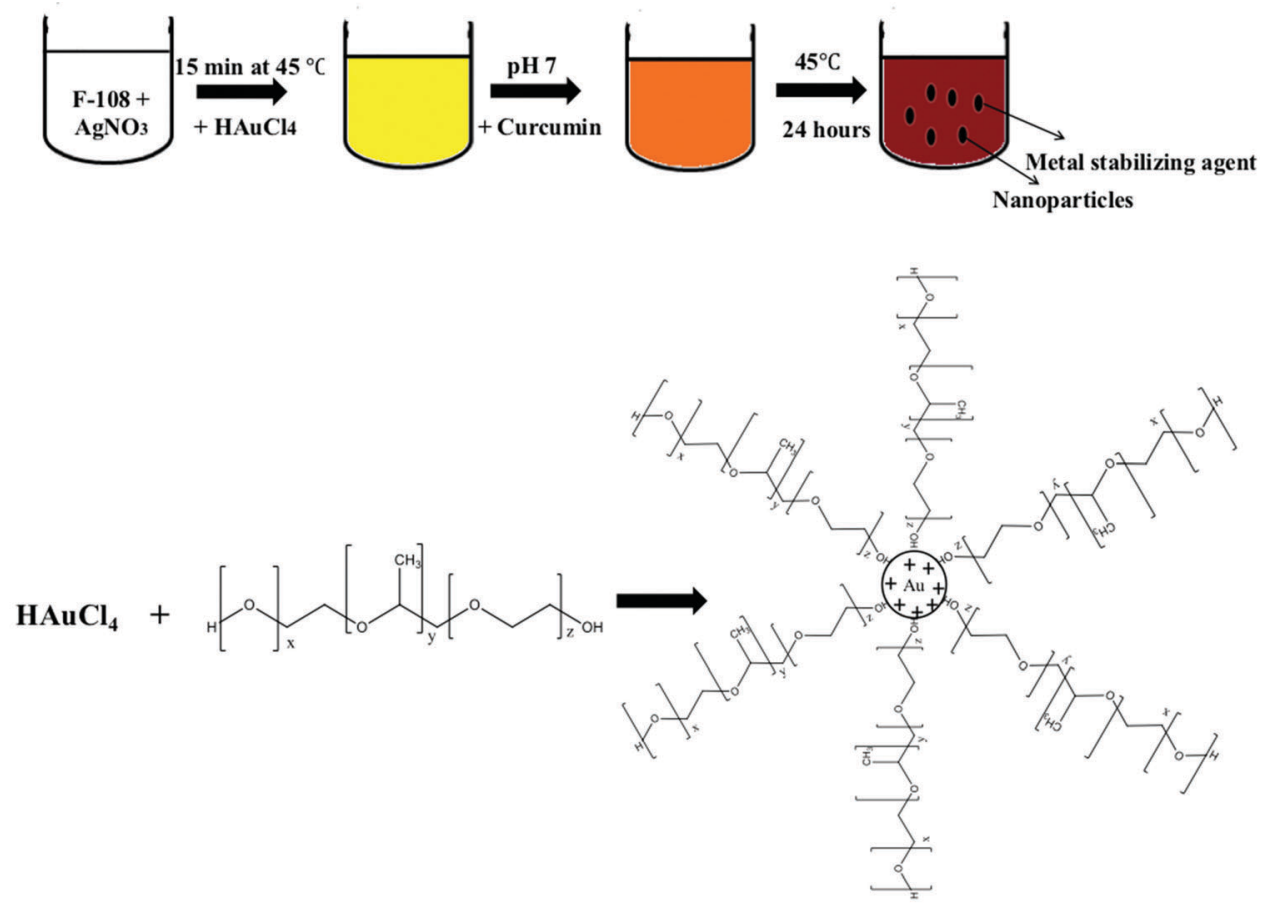

B

RRS Signal

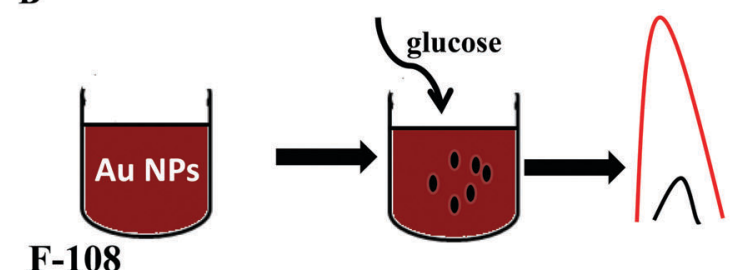

F-108

Functionalization
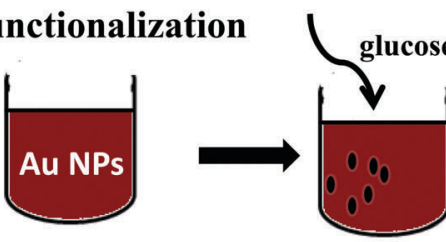

RRS Signal

CTAB

Functionalization

Scheme 1 (A) Scheme of the general green synthetic procedure during the preparation of gold particles by reducing $\mathrm{Au}^{3+}$ using curcumin in polymeric media; (B) illustration of the high RRS signal of Au NPs in the presence of glucose.

concentration to $500 \mu \mathrm{M}$ as shown in Fig. 1A. Further decreasing the concentration to below $500 \mu \mathrm{M}$ did not have any remarkable effect indicating the inertness of the particle size in this concentration range.

Resonance Rayleigh Scattering (RRS) spectra were measured by applying synchronous fluorescence spectroscopy by keeping the wavelength interval $(\Delta \lambda)$ at $0 \mathrm{~nm} .{ }^{30}$ The RRS spectra of F-108 conjugated Au NPs prepared at different polymer concentrations are depicted in Fig. 1B. The formed gold nanoparticles gave two major localized SPR peaks, at $\sim 435 \mathrm{~nm}$ and at $\sim 550 \mathrm{~nm}$ with a minor peak at $\sim 385 \mathrm{~nm}$. However, for the particles prepared at low concentrations, $\sim 50 \mu \mathrm{M}$, the intensity of the peaks was enhanced, compared to the particles prepared at a high concentration of above $50 \mu \mathrm{M}$. To determine the emission spectrum, the Au NPs were excited at two absorption wavelengths: $425 \mathrm{~nm}$ for curcumin and $500 \mathrm{~nm}$ for Au NPs. The emission spectra at both excitation wavelengths $\left(\lambda_{\text {ex }}=425 \mathrm{~nm}\right.$ and $\lambda_{\mathrm{ex}}=500 \mathrm{~nm}$ ) of the Au NPs prepared in four different concentrations of F-108 are shown in Fig. 1C and D respectively. The emission spectra of the Au NPs prepared at four different concentrations of F-108 gave an emission peak at $\sim 540 \mathrm{~nm}$ and a band at $\sim 670 \mathrm{~nm}$ for both the excitation wavelengths. The peak at $\sim 540 \mathrm{~nm}$ is most likely due to emission from the ligand, curcumin, associated with Au NPs whereas the band at 

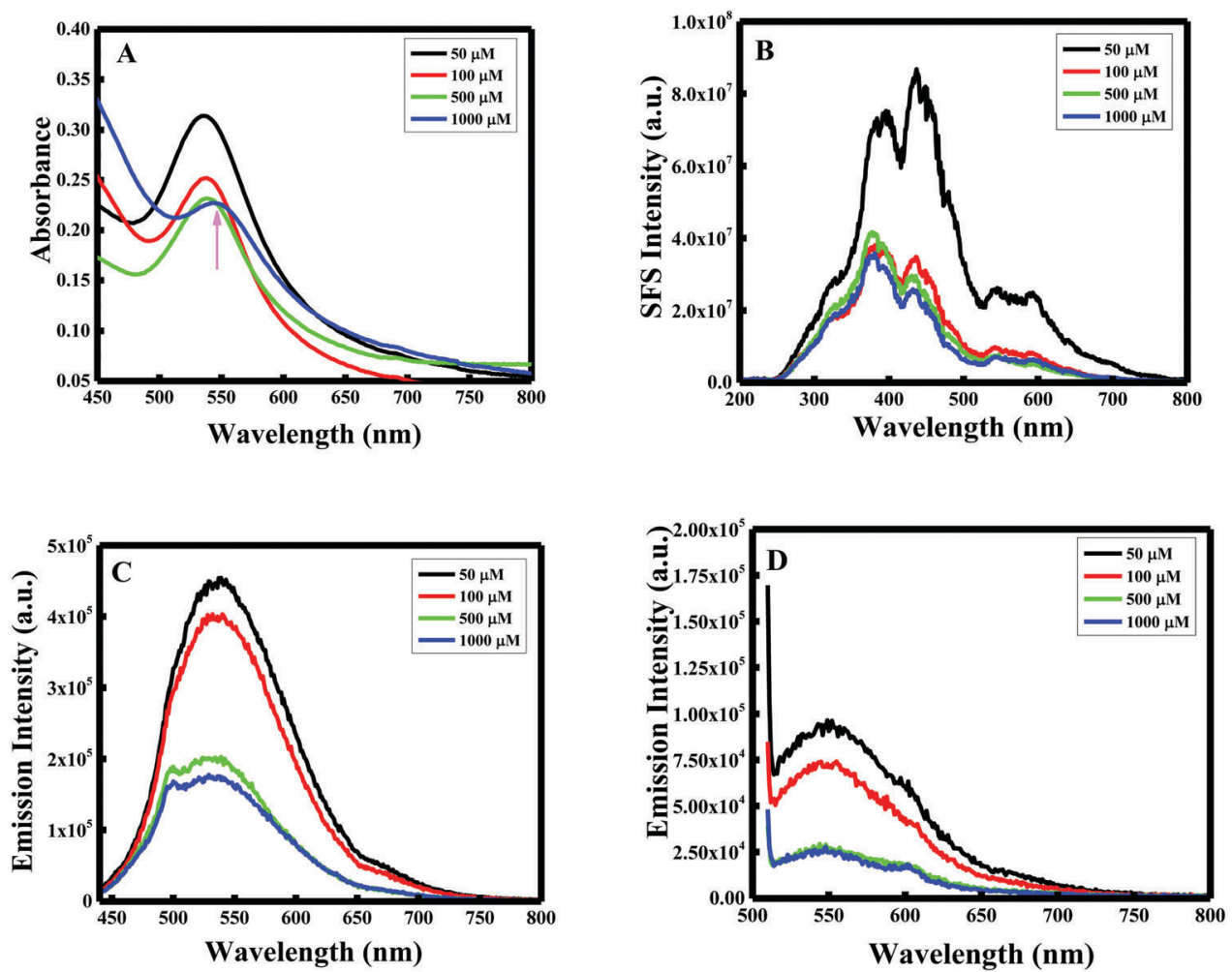

Fig. 1 (A) UV-Vis spectra, (B) synchronous fluorescence spectra at $\Delta \lambda=0 \mathrm{~nm}$, (C) fluorescence emission spectrum excited at $\lambda=425 \mathrm{~nm}$ and (D) fluorescence emission spectrum excited at $\lambda=500 \mathrm{~nm}$, of F-108 and curcumin functionalized Au nanoparticles prepared at different concentrations of the polymer.

$\sim 670 \mathrm{~nm}$ suggests that of Au NPs. Interestingly, the emission intensity of the Au NPs decreased as the concertation of F-108 increased during preparation, which is due to the decrease in the absorbance of the Au NPs with an increase in the F-108 concentration. This change in the fluorescence intensity/RRS intensity as a consequence of change in the absorbance value is the result of alteration in the particle size and/or shape (though a minor contribution of the inner filter effect from the absorption of curcumin at $\sim 450 \mathrm{~nm}$ cannot be ruled out), which could be easily verified in the SEM images depicted in Fig. 2. The particle size distribution was in the range of $10-30 \mathrm{~nm}$. The biggest spheres with a size between 20 and $30 \mathrm{~nm}$, with some heterogeneous distribution, were obtained for Au NPs prepared using $1 \mathrm{mM}$ of F-108 and for the other three F-108 concentrations the size of the Au NPs was between 10 and 20, which also justifies the obtained shift in the UV-Vis spectra.

\subsection{Effect of curcumin concentration}

The effect of curcumin concentration on the formation of $\mathrm{Au}$ NPs was not regular. In fact, while increasing the concentration of curcumin during the synthesis of Au NPs, the localized SPR spectrum showed a blue shift with an increase in the intensity until $10 \mathrm{mM}$ of curcumin, while for $50 \mathrm{mM}$ the intensity
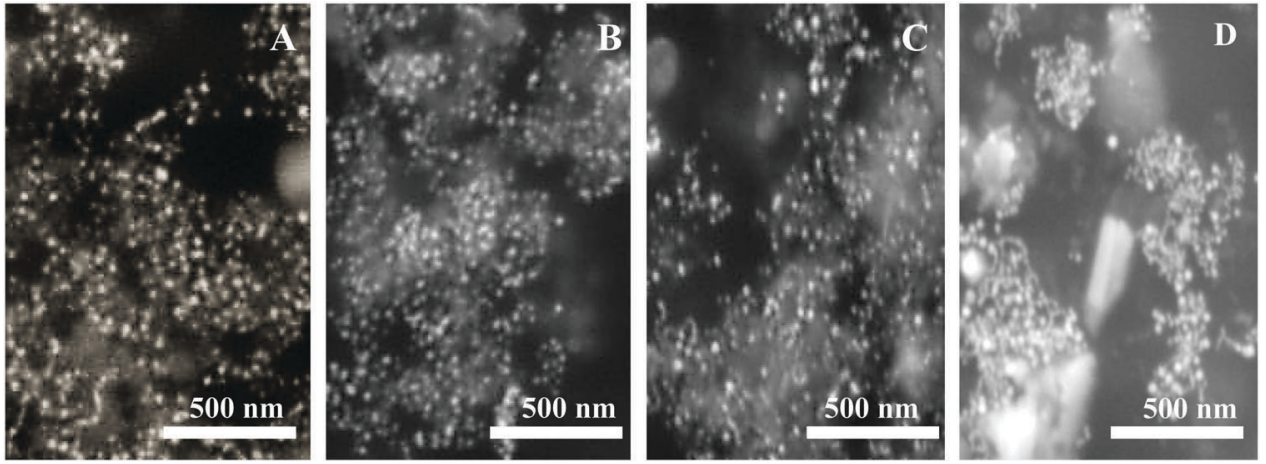

Fig. 2 SEM images of F-108 and curcumin functionalized gold nanoparticles prepared at different concentrations of the polymer: $(A) C=50 \mu M$; (B) $C=100 \mu \mathrm{M}$; (C) $C=500 \mu \mathrm{M}$ and (D) $C=1000 \mu \mathrm{M}$. 

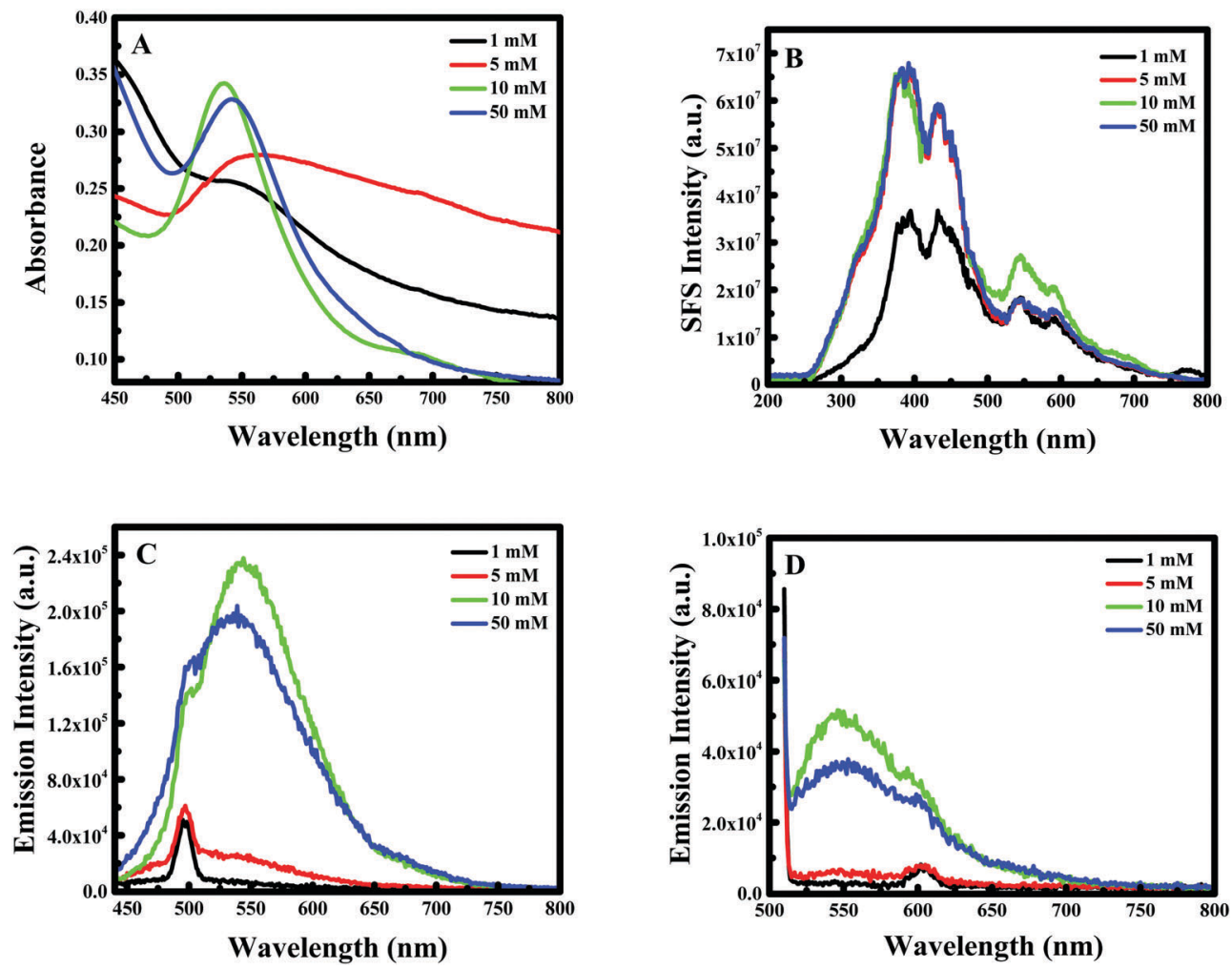

Fig. 3 (A) UV-Vis spectra, (B) synchronous fluorescence spectra at $\Delta \lambda=0 \mathrm{~nm}$, (C) fluorescence emission spectrum excited at $\lambda=425 \mathrm{~nm}$ and (D) fluorescence emission spectrum excited at $\lambda=500 \mathrm{~nm}$, of F-108 and curcumin functionalized Au nanoparticles prepared at different concentrations of curcumin.

decreased again with a red shift for the peak as shown in Fig. 3A.

For example, when the concentration of curcumin was equal to $1 \mathrm{mM}$ a clear peak was obtained at $\lambda=567 \mathrm{~nm}$, which shifted to $\lambda=535 \mathrm{~nm}$ on increasing the concentration to $10 \mathrm{mM}$; nevertheless, the peak then shifted back to $\lambda=544 \mathrm{~nm}$ when the concentration of curcumin was further increased to $50 \mathrm{mM}$. The RRS spectra of F-108 conjugated Au NPs prepared at different curcumin concentrations were slightly different, as represented in Fig. 3B. For instance, when varying the curcumin concentration, the nanogold particles prepared at the four concentrations of curcumin gave two major localized SPR peaks at $\sim 380 \mathrm{~nm}$ and at $\sim 550 \mathrm{~nm}$ with a minor peak at $\sim 435 \mathrm{~nm}$. Initially, increasing the concentration of curcumin did not influence the peak positions but enhanced the peak intensity, which saturated at a higher concentration of curcumin used. The fluorescence emission spectra at both excitation wavelengths, $\lambda_{\mathrm{ex}}=425 \mathrm{~nm}$ and $\lambda_{\mathrm{ex}}=500 \mathrm{~nm}$, of Au NPs prepared at four different concentrations of curcumin are compared in Fig. 3C \& D respectively. The spectra did not show any appreciable change in the peak position on changing either the curcumin concertation or F-108 concentration (discussed earlier). However, when a low concentration of curcumin $(1 \mathrm{mM}$ and $5 \mathrm{mM}$ ) was used for the preparation of $\mathrm{Au}$ NPs, the obtained fluorescence intensity was low, which probably suggests the presence of a lower amount of curcumin at the nanoparticle surfaces and/or formation of different sizes/shapes of NPs at a lower concentration of curcumin, which was later on confirmed from SEM images depicted in Fig. 4.

Accordingly, $10 \mathrm{mM}$ of curcumin was sufficient to boost the intensity and obtain satisfactory results. Interestingly, for $10 \mathrm{mM}$ of curcumin, small and uniform spheres were obtained with a size between 10 and $20 \mathrm{~nm}$. However, at $1 \mathrm{mM}$ and $5 \mathrm{mM}$ curcumin concentrations, different shapes of gold nanoparticles were obtained with a size between 120 and $140 \mathrm{~nm}$ and between 100 and $120 \mathrm{~nm}$, respectively. The decrease in the particle size may be due to the increase in the density of the functional groups which stabilize smaller gold nanoparticles. Thus, at $50 \mathrm{mM}$ of curcumin, the particle size increases again with the size between 30 and $40 \mathrm{~nm}$. This increase is due to the poor solubility of curcumin at neutral $\mathrm{pH}$, where the curcumin molecule will precipitate in the media inhibiting the formation of smaller $\mathrm{Au}$ NPs. To verify the formation of $\mathrm{Au}$ NPs, EDX analysis was carried out. The EDX spectrum demonstrated the presence of $\mathrm{Au}$ peaks and carbon peaks as expected due to capping of F-108/curcumin (see Fig. 5A-D and 6A-D).

\subsection{Role of $\mathrm{Ag}^{+}$ions}

Silver ions $\left(\mathrm{Ag}^{+}\right)$are known to bind preferentially onto gold facets, which can result in anisotropic crystal growth through the well-known under potential deposition mechanism. ${ }^{49}$ Therefore, to understand the role of silver nitrate in our synthesis, an experiment was performed using the optimal concentrations of F-108 and curcumin obtained from different experiments. 

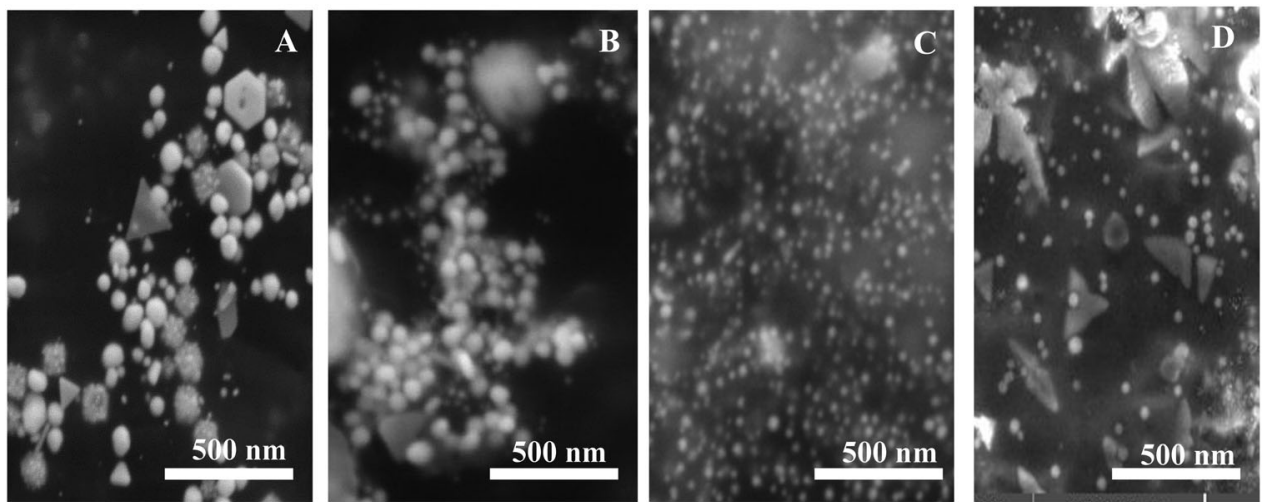

Fig. 4 SEM images of F-108 and curcumin functionalized gold nanoparticles prepared at different concentrations of curcumin: (A) $C=1 \mathrm{mM}$; (B) $C=5 \mathrm{mM}$; (C) $C=10 \mathrm{mM}$ and (D) $C=50 \mathrm{mM}$.
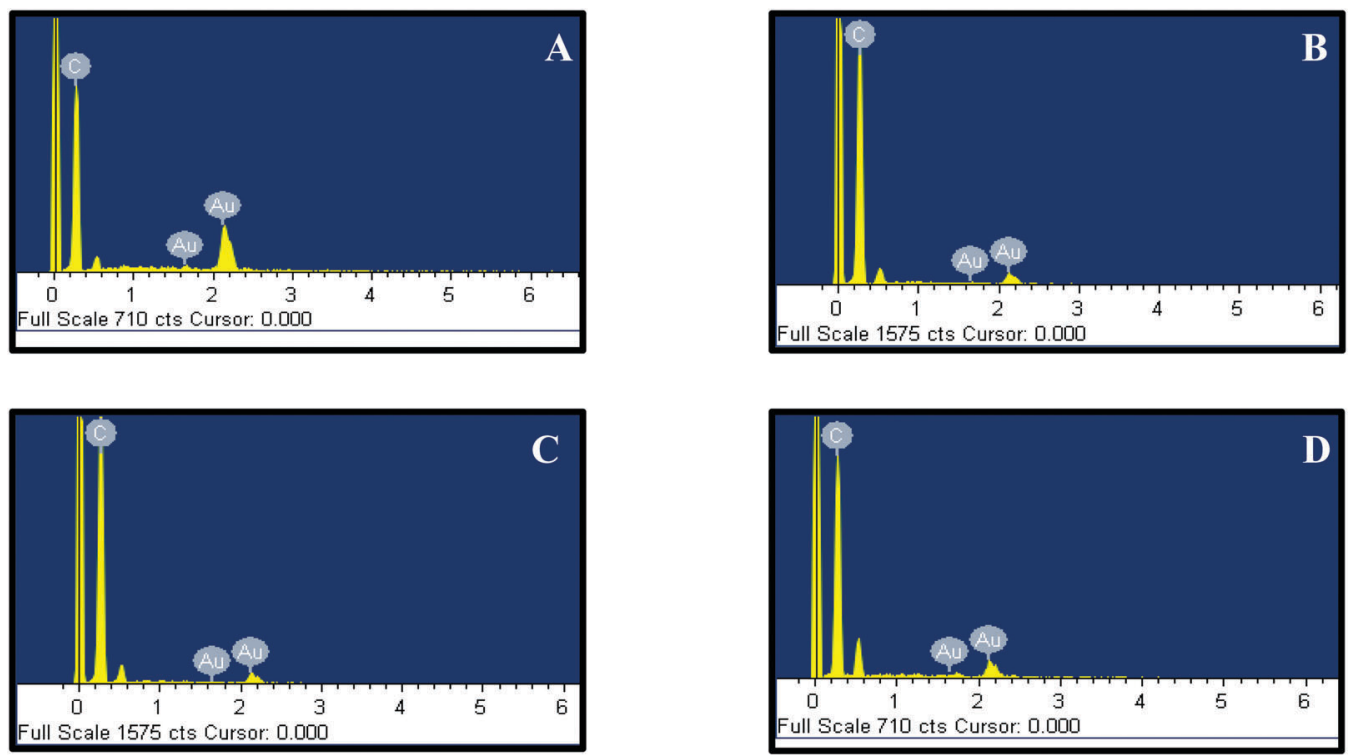

Fig. 5 EDX of F-108 and curcumin functionalized gold nanoparticles prepared at different concentrations of the polymer: $(A) C=50 \mu M$; $(B) C=100 \mu M$; (C) $C=500 \mu \mathrm{M}$ and (D) $C=1000 \mu \mathrm{M}$

The higher absorption intensity as well as uniform particle sizes and shapes were obtained for the F-108 concentration of $\sim 50 \mu \mathrm{M}$ and the curcumin concertation of $10 \mathrm{mM}$, thus, these concentrations were chosen for further investigation. The localized SPR spectrum showed a blue shift with an increase in the intensity when $\mathrm{Ag}^{+}$ions were added during the preparation procedure. For example, in the presence of $\mathrm{AgNO}_{3}$ a clear peak was obtained at $536 \mathrm{~nm}$ (as shown in Fig. 7A) that shifted to $550 \mathrm{~nm}$ in the absence of $\mathrm{AgNO}_{3}$. The RRS spectra of F-108 conjugated Au NPS prepared in the presence and absence of $\mathrm{AgNO}_{3}$ is depicted in Fig. 7B.

The gold nanoparticles prepared in the two experiments gave three major localized SPR peaks at $\sim 385 \mathrm{~nm}, \sim 435 \mathrm{~nm}$ and $\sim 550 \mathrm{~nm}$. However, for the particles prepared without $\mathrm{AgNO}_{3}$ the intensity of the peak at $550 \mathrm{~nm}$ was enhanced, compared to the particles prepared in the presence of $\mathrm{AgNO}_{3}$. The emission spectra at both excitation wavelengths $\lambda_{\mathrm{ex}}=425 \mathrm{~nm}$ and $\lambda_{\mathrm{ex}}=500 \mathrm{~nm}$ (as presented in Fig. 7C and D respectively) of the $\mathrm{Au}$ NPs obtained for the two experiments are similar and the peak positions did not show any appreciable change except that the fluorescence intensity for the Au NPs in the absence of $\mathrm{AgNO}_{3}$ was enhanced compared to the AuNPs formed in the presence of $\mathrm{AgNO}_{3}$. The increase in the intensity cannot be only due to the change in the particle size/shape of the $\mathrm{Au}$ NPs because the absorbance in the presence of $\mathrm{Ag}^{+}$was higher than in the absence of $\mathrm{Ag}^{+}$ions. Thus, the enhancement in fluorescence as well as the RRS intensity is not solely due to the change in the shape/size of the Au NPs, rather it is a combination of the influence of the shape/size of the Au NPs as well as the extent of curcumin conjugation at the interface of the Au NP surface and F-108. The difference in size/shape could be easily verified in the SEM images as shown in Fig. 8. In the presence of $\mathrm{AgNO}_{3}$ uniform spherical particles were obtained (Fig. 8A) with a size between 10 and $20 \mathrm{~nm}$, whereas in the 

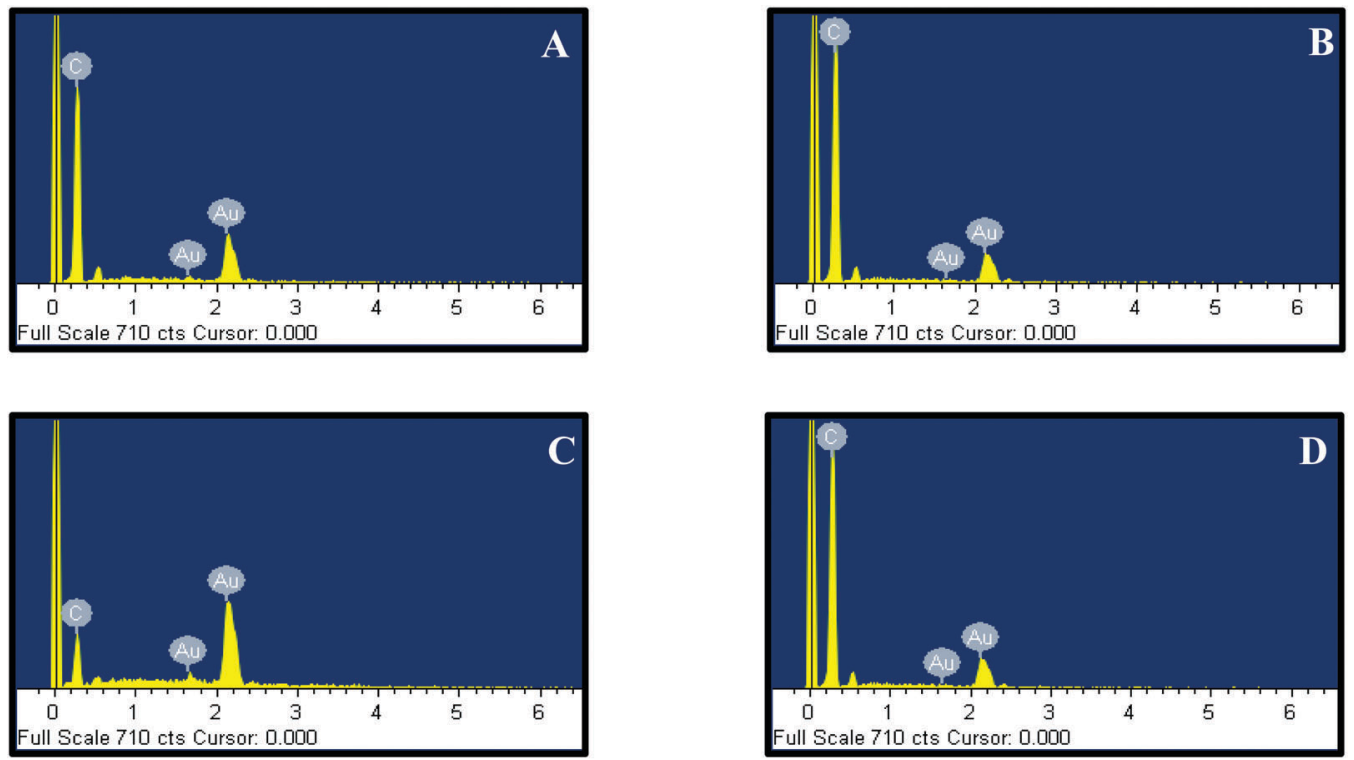

Fig. 6 EDX of F-108 and curcumin functionalized gold nanoparticles prepared at different concentrations of curcumin: (A) $C=1 \mathrm{mM}$; (B) $C=5 \mathrm{mM}$; (C) $C=10 \mathrm{mM}$ and (D) $C=50 \mathrm{mM}$.
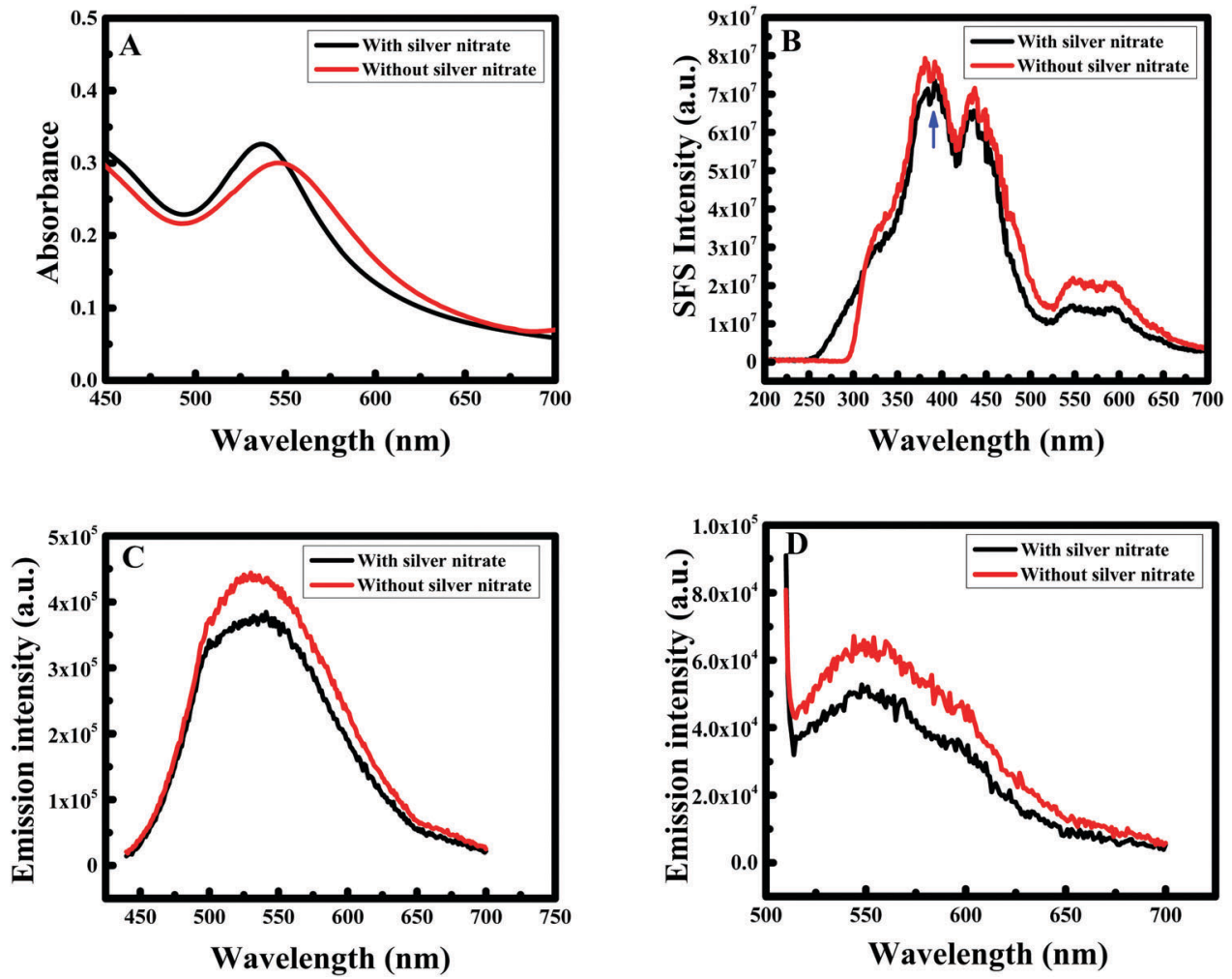

Fig. 7 (A) UV-Vis spectra, (B) synchronous fluorescence spectra at $\Delta \lambda=0 \mathrm{~nm}$, (C) fluorescence emission spectrum excited at $\lambda=425 \mathrm{~nm}$ and (D) fluorescence emission spectrum excited at $\lambda=500 \mathrm{~nm}$, of F-108 and curcumin functionalized Au nanoparticles prepared with/without AgNO $\mathrm{H}_{3}$

absence of $\mathrm{AgNO}_{3}$ a few rod-shaped particles were formed (see Fig. 8B) in addition to the spherical particles with an increase in the particle size between 20 and $30 \mathrm{~nm}$.

Hence, the mixture of gold nanoparticles and nanorods has a moderate and optimally enhanced RRS signal. The increase in the florescence intensity could be due to the higher extent of curcumin conjugation in the Au NPs prepared in the presence of $\mathrm{AgNO}_{3}$. EDX analysis (Fig. 8C and D) also did not show any peak for $\mathrm{Ag}$ concluding that silver nanoparticles were not formed during the formation of Au NPs and demonstrating 

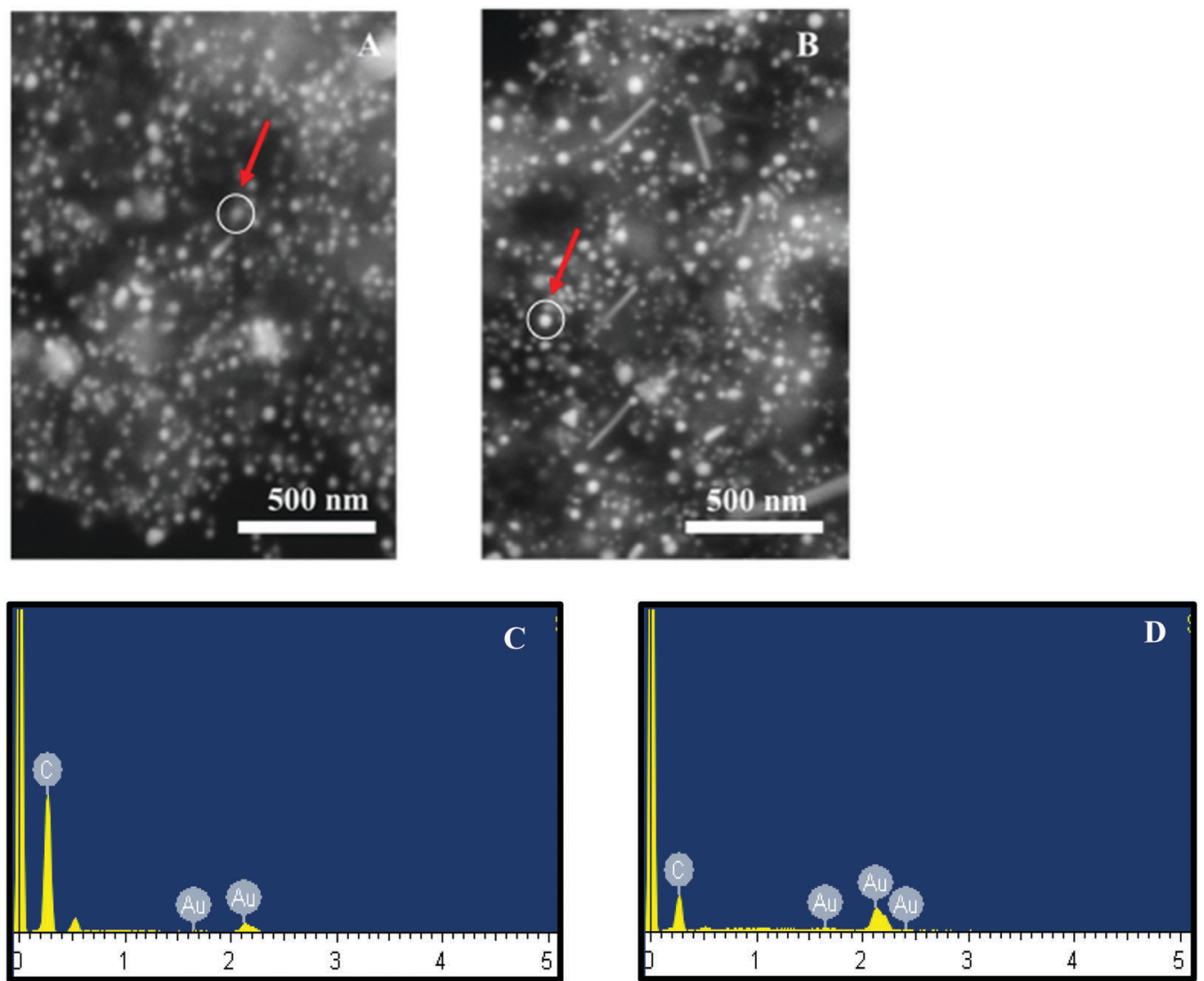

Fig. 8 SEM images of F-108 and curcumin functionalized gold nanoparticles prepared with silver nitrate (A) and without silver nitrate (B). Corresponding EDX is given in (C) for with silver nitrate and in (D) for without silver nitrate. The arrow mark indicates the area of analysis.

that the role of silver nitrate was only to facilitate the formation of uniform Au NPs and it is used for tuning the aspect ratio of the formed nanogold.

To further establish the characteristics of Au NPs, the gold nanoparticles prepared with/without $\mathrm{AgNO}_{3}$ were analyzed using the X-ray diffraction (XRD) technique (see Fig. 9). The X-ray diffractograms were similar for the Au NPs prepared in both

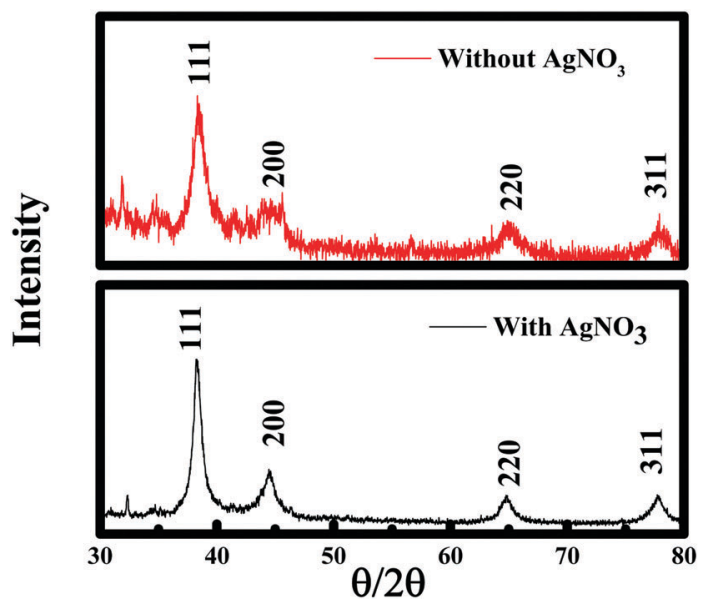

Fig. 9 X-ray diffraction pattern of curcumin conjugated gold nanoparticles and nanowires prepared with and without silver nitrate, respectively. cases. The characteristic peaks at $38.269^{\circ}, 44.600^{\circ}, 64.678^{\circ}$, and $77.549^{\circ}$ are assigned to the (111), (200), (220), and (311) reflections of the face centered cubic unit cell, which are typical for $\mathrm{Au}$ particles. ${ }^{25,26,50}$ The difference in the peak intensity suggests the highly crystalline nature of the Au NPs prepared in the presence of silver nitrate compared to the Au NPs formed without $\mathrm{AgNO}_{3}$, which could be due to the variation in shape. However, the peaks between $15^{\circ}-30^{\circ}$ correspond to the curcumin molecule. Thermogravimetric analysis showed the decomposition of curcumin and $\mathrm{AgNO}_{3}$ at $\sim 400{ }^{\circ} \mathrm{C}^{51}$ and $\sim 470{ }^{\circ} \mathrm{C}^{52}$ respectively as given in Fig. 10, which is consistent with other reported values. TGA data of the Au NPs with/without $\mathrm{AgNO}_{3}$ illustrated a similar degradation pattern. Both of them demonstrated a significant weight loss of $\sim 50 \%$ at $\sim 230{ }^{\circ} \mathrm{C}$ indicating that both Au NPs are capped with $\sim 50 \%$ of curcumin and F-108. ${ }^{53}$ Interestingly, no loss of water was observed around $100{ }^{\circ} \mathrm{C}$ and it can be assumed that the samples are dehydrated, thus, the nanoparticles that were formed are stable. ${ }^{9}$ Curcumin and F-108 polymer conjugation/ capping with AuNPs was further established by spectroscopic analysis. The FT-IR spectra for Au NPs prepared with/without $\mathrm{AgNO}_{3}$ and pure curcumin are exhibited in Fig. 11. In the FT-IR spectrum of curcumin, the strong peak at $1630 \mathrm{~cm}^{-1}$ has a predominantly mixed $\nu(\mathrm{C}-\mathrm{C})$ and $\nu(\mathrm{C}=\mathrm{O})$ character. Another strong band at $1601 \mathrm{~cm}^{-1}$ is attributed to the symmetric aromatic ring stretching vibrations $\nu\left(\mathrm{C}_{\text {ring }}\right)$. The $1519 \mathrm{~cm}^{-1}$ peak is assigned to $(\mathrm{C}=\mathrm{O})$. An enol $\mathrm{C}-\mathrm{O}$ peak was obtained at $1262 \mathrm{~cm}^{-1}$, 


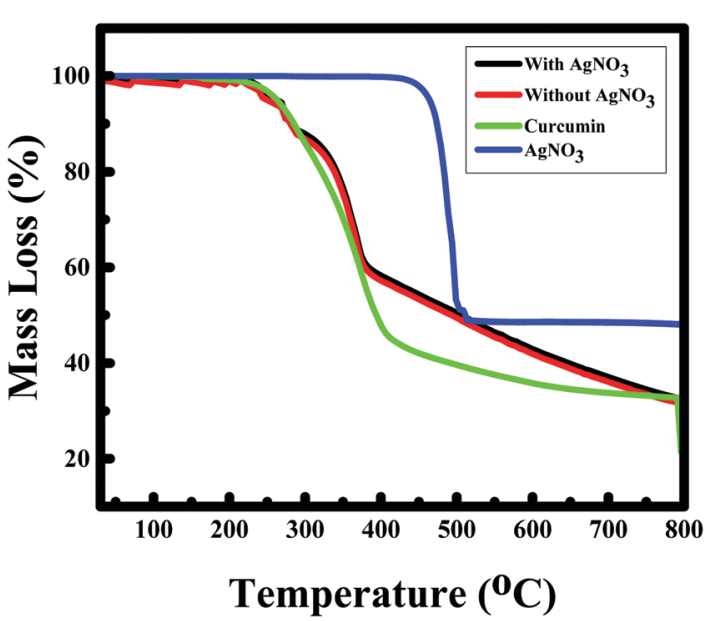

Fig. 10 TGA of curcumin functionalized gold nanoparticles (Au NPs) with and without silver nitrate, and curcumin and silver nitrate, respectively.

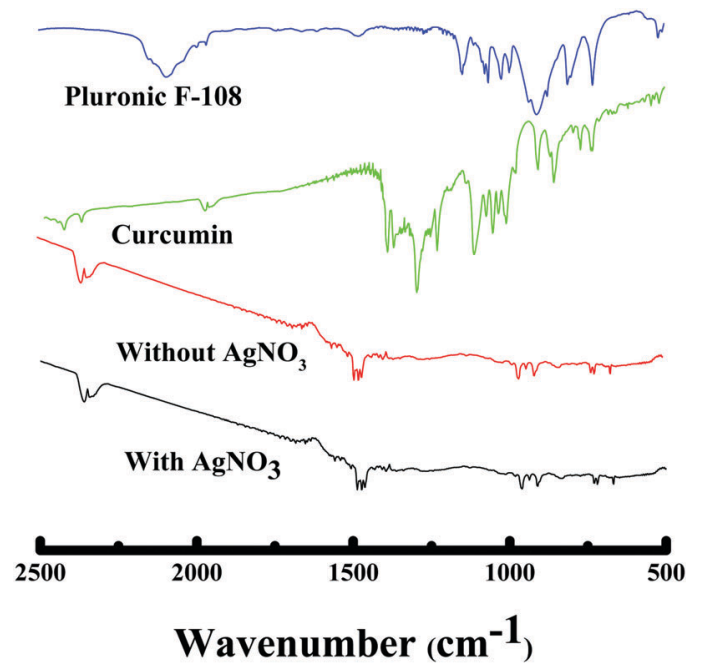

Fig. 11 FT-IR spectra of curcumin functionalized gold nanoparticles (Au NPs) with/without silver nitrate and curcumin, respectively.

a $\mathrm{C}-\mathrm{O}-\mathrm{C}$ peak at $1018 \mathrm{~cm}^{-1}$, benzoate trans-CH vibration at $978 \mathrm{~cm}^{-1}$ and cis $\mathrm{CH}$ vibration of an aromatic ring at $703 \mathrm{~cm}^{-1}$. The bands at $1404 \mathrm{~cm}^{-1}$ represent the in-plane bending of the hydroxyl groups of the phenolic group. All the three bands (1404, 1262 and $978 \mathrm{~cm}^{-1}$ ) were completely absent in the FT-IR spectrum of $\mathrm{Au}$ NPs prepared with and without $\mathrm{AgNO}_{3}$, which suggests the interaction of $\mathrm{HAuCl}_{4}$ at these sites. The bands at $774 \mathrm{~cm}^{-1}$ and $1424 \mathrm{~cm}^{-1}$ correspond to the olefinic in-plane bending vibrations of the heptadiene chain of curcumin. ${ }^{54}$ The peaks at $2890 \mathrm{~cm}^{-1}, 1476 \mathrm{~cm}^{-1}$ and $1110 \mathrm{~cm}^{-1}$, corresponding to asymmetric stretching vibrations of $-\mathrm{O}-\mathrm{H},-\mathrm{C}-\mathrm{H}, \mathrm{C}-\mathrm{C}$ and $\mathrm{C}=\mathrm{O}$ of pluronic F-108 were also missing in the FT-IR spectrum of the Au NPs.

\subsection{Estimation of glucose from the change in the RRS signal of the Au NPs}

Biofunctionalized nanomaterials can be used as carriers or tracers to obtain an amplified detection signal and stabilized recognition probes. Nanomaterials are well known to possess excellent electrical, optical, thermal, and catalytic properties and strong mechanical strength producing a synergic effect, resulting in significant signal amplification which offers great opportunities to construct nanomaterial-based sensors. ${ }^{55}$ The field of sensors encompasses a wide variety of materials and devices used for capturing physical, chemical or biological stimuli converting them to measurable output signals. ${ }^{55}$ Gold nanoparticles have been introduced as carriers for signal amplification and shown to promote the direct electron transfer between the biomolecules and the electrode surface and have also demonstrated their advantages in bioanalysis using localized SPR transduction. This method is usually based on the change of the dielectric constant of the propagating surface plasmon environment of gold particles where the detection of the analyte can be recorded in different ways like the changes of the angle, intensity, or phase of the reflected light. In fact, the surface plasmons on gold nanoparticles provoke a perturbation of the evanescent field of the gold film in addition to the immobilized bioreceptor unit and the recognized analyte. ${ }^{56}$ Gold nanoparticles exhibit localized SPR at specific incident wavelengths, generating strong light scattering and the appearance of intense surface plasmon absorption bands. ${ }^{57}$ Using this phenomenon many localized SPR-based chemical and biological sensors have been developed. Since, uniform gold nanoparticles were obtained while adding silver nitrate with $10 \mathrm{mM}$ of curcumin and $50 \mu \mathrm{M}$ of the F-108 polymer, $\mathrm{Au}$ nanoparticles prepared under these conditions were chosen for glucose sensing. When glucose was added in a high concertation to a solution of Au NPs, there was no remarkable change in the localized SPR band, which was confirmed by the fluorescence emission spectra. Interestingly, the addition of glucose on the other hand had a remarkable effect on the RRS signal of Au NPs, about 8-fold increase in the RRS intensity was observed, as shown in Fig. 12A, without changing the spectral shape and RRS wavelength maximum. This is mainly due to the large surface area and the high surface free energy of nanoparticles, which can make the glucose molecule inevitably be adsorbed on the surface of the nanoparticles. This phenomenon could be the consequence of multivalent binding with the polymer, which forms larger aggregates. We suspect that glucose is not directly involved in binding to the surface of Au NPs, thus, no change in the fluorescence emission intensity (therefore, in localized SPR absorbance) was observed. In fact, the polymer present in the system competitively binds with the glucose molecule to form large aggregates. The resonance Rayleigh scattering intensity is sensitive to particle size/aggregation, and thus, the presence of glucose in the solution helps in creating aggregates and boosts the RRS intensity. To further verify our hypothesis and the role of F-108 in glucose sensing, Au NPs were functionalized with CTAB using curcumin. These CTAB functionalized Au NPs were later on applied to detect glucose molecules. As summarized in Table 1, the CTAB functionalized Au NPs did not show any significant change in the RRS intensity in the presence of glucose, whereas F-108 capped Au NPs remarkably enhanced the RRS signal reconfirming the role of the polymer in associating with the glucose molecules. 

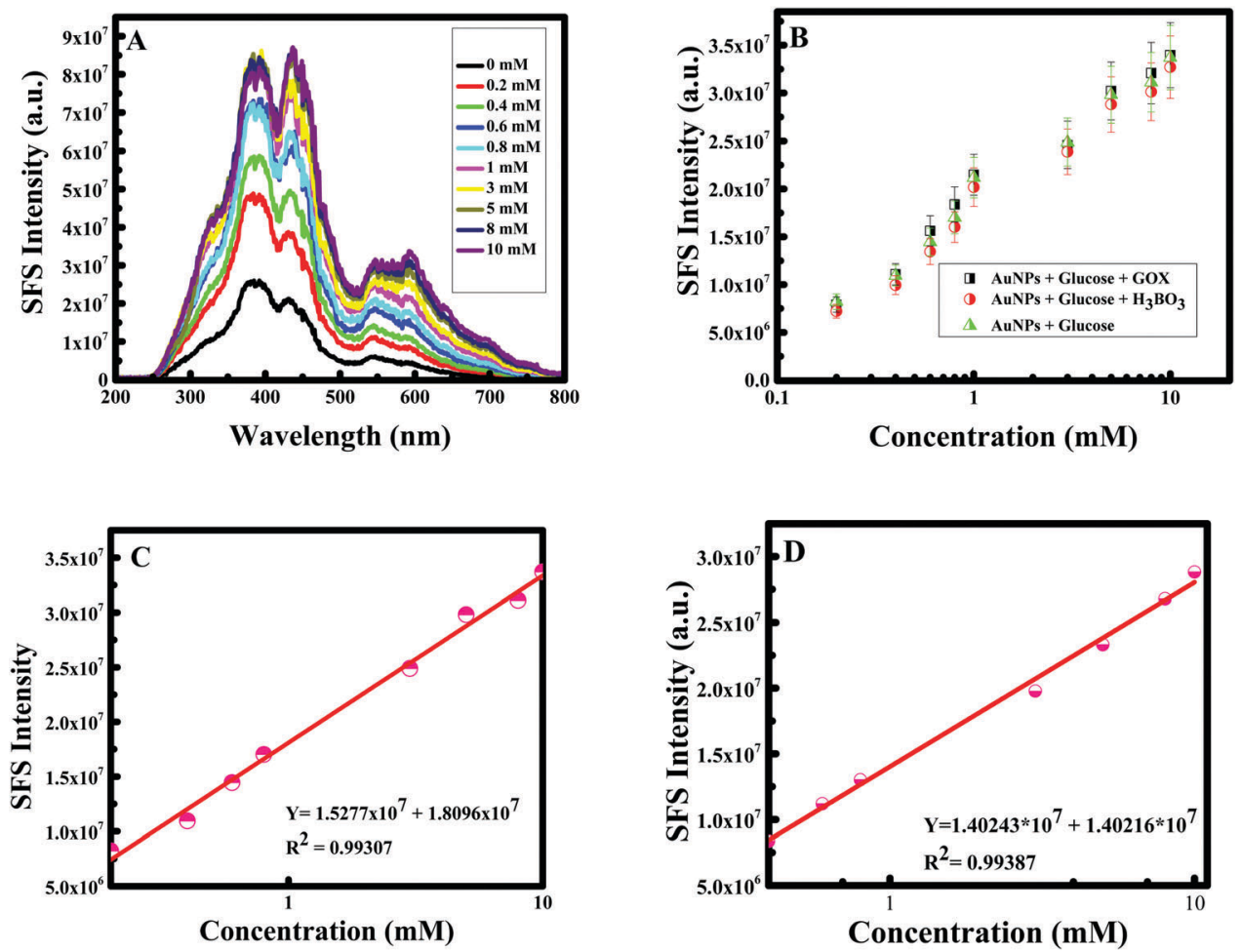

Fig. 12 (A) Synchronous fluorescence spectra (SFS) at $\Delta \lambda=0 \mathrm{~nm}$ of curcumin functionalized Au NPs with different concentrations of glucose; (B) calibration curve for Au NPs + glucose + GOx, Au NPs + boric acid + glucose, and AuNPs + glucose; (C) linear correlation of the SFS intensity of Au NPs vs. concentration of glucose; (D) linear correlation of the SFS intensity of Au NPs vs. concentration of glucose spiked in a real sample.

Table 1 Change in the $/ / I_{0}$ value during glucose sensing using F-108 functionalized Au NPs vs. CTAB functionalized Au NPs

\begin{tabular}{lll}
\hline & $I / I_{0}$ & \\
\cline { 2 - 3 } Concentration (in $\mathrm{mM})$ & Polymer (F-108) & CTAB \\
\hline 0 & 1.0 & 1.0 \\
0.2 & 1.8 & 1.0 \\
0.6 & 3.2 & 1.0 \\
5 & 6.6 & 1.0
\end{tabular}

Note: $I$ and $I_{0}$ are the RRS intensity of Au NPs in the presence and absence of glucose.

Gold nanoparticles are biocompatible; ${ }^{4}$ as a consequence, gold can act as the immobilization enzyme matrix, and quickly enhance the activity between the nanoparticles and glucose molecules. Hence, glucose sensing was established using boric acid and glucose oxidase to verify the efficiency of gold nanoparticles. Generally, boric acid-containing hydrogels are important intelligent materials and the introduction of boric acid functionality to these hydrogels allows them to exhibit many interesting properties, such as glucose-selectivity/sensitivity, reversible binding and self-healing ability ${ }^{58}$ and glucose oxidase is identified as an oxidoreductase enzyme that catalyzes and facilitates the oxidation of glucose and as a consequence the detection of glucose molecules. ${ }^{59}$ Comprehending the significance of Au NPs for selective sensing of glucose, a calibration curve was made by measuring the RRS single of F-108 capped Au NPs in the presence of boric acid as well as glucose oxidase in different concentrations of glucose. The RRS signal alteration of Au NPs with glucose concentration is plotted in Fig. 12B. The RRS spectra of $\mathrm{Au}$ NPs in the presence of different concentrations of glucose in 3 different cases showed similar behaviour: a continuous and remarkable increase in the shape of the RRS spectra of the Au NPs. Hence, the sensing of glucose using F-108 capped gold nanoparticles as probes can be done without any supplement enzyme. The linear correlation of RRS signal alteration of Au NPs vs. glucose concentration is plotted in Fig. 12C. As can be seen in the plot, in a range of concentrations between 0 - and $10 \mathrm{mM}$, the curve showed a linear change. This linear increase was very well fitted with a linear equation, $Y=1.5277 \times$ $10^{6}+1.8096 \times 10^{7}$ with $R^{2}=0.99307$. Such a good correlation justifies the applicability of the present method for the determination of glucose in the given concentration range. As the gold nanoparticles were able to detect the standard solution of glucose we were interested to detect a real sample of glucose. Human blood serum samples were collected from patients in the American University of Beirut Medical Center to test the capacity of curcumin mediated F-108 functionalized gold nanoparticles to detect glucose present in the serum. In this case, several solutions were prepared by adding $30 \mu \mathrm{L}$ of serum with a known glucose concentration in the range between 0 and $10 \mathrm{mM}$. The calibration curve of curcumin mediated F-108 functionalized Au NPs at different concentrations of glucose using the RRS signal is depicted in Fig. 12D. Excitingly, as expected, increasing the glucose concentration induced an increase in the RRS intensity. To test the selectivity, other potential anti-oxidant interferences 


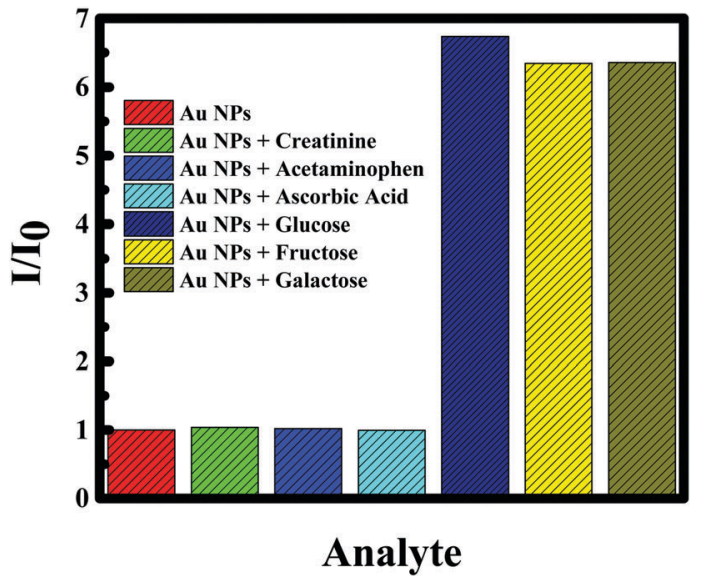

Fig. 13 Ratio of the SFS intensity $\left(/ / /_{0}\right)$ of Au NPs for different species in the absence of antioxidant, creatinine, acetaminophen, ascorbic acid, glucose, fructose and galactose.

Table 2 Recovery results of the method

\begin{tabular}{lcll}
\hline & $\begin{array}{l}\text { Theoretical } \\
\text { concentration } \\
\left(\mathrm{mmol} \mathrm{L}^{-1}\right)\end{array}$ & $\begin{array}{l}\text { Experimental } \\
\text { concentration } \\
\left(\mathrm{mmol} \mathrm{L}^{-1}\right)\end{array}$ & $\begin{array}{l}\text { Recovery } \\
(\%)\end{array}$ \\
\hline Unknown 1 & 0.80 & 0.83 & 109 \\
Unknown 2 & 8.0 & 8.7 & 104 \\
Unknown 3 & 10.0 & 10.5 & 105 \\
\hline
\end{tabular}

from various substances were tested. Creatinine, acetaminophen and ascorbic could interfere during the estimation of glucose. As confirmed from Fig. 13, $7 \mathrm{mM}$ of creatinine or acetaminophen or ascorbic acid did not alter the RRS signal of Au NPs unlike the $\sim 7$-fold increase observed for the same concertation of glucose. However, as for fructose and galactose, an alteration of the RRS signal was observed and this could be explained by the fact that these two compounds are glucose isomers. Hence, the response of the Au NPs with glucose is similar to the one obtained in the presence of fructose and galactose. The analytical recovery of real samples using the proposed method was studied by testing

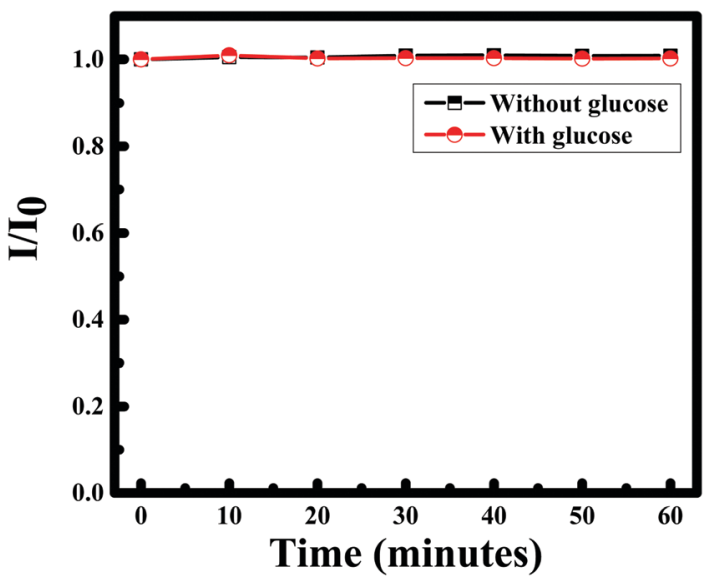

Fig. 14 Plot of $/ / I_{0}$ of curcumin functionalized Au nanoparticles with time in the absence and presence of glucose.
3 serum samples. The results obtained are summarized in Table 2. The percent of glucose recovery of our method was found to be between 100 and $110 \% \pm 2.9$ (mean \pm S. D.) which is quite acceptable. Finally, the photostability of the curcumin mediated F-108 functionalized Au NPs was verified, which is illustrated in Fig. 14. When the RRS signal of the Au NPs was recorded in the absence and presence of glucose for one hour, the signal was found to be remarkably stable indicating that the current sensor is quite stable during the measurement time.

\section{Conclusion}

We have shown a facile synthetic procedure for the preparation of uniform F-108 functionalized Au NPs in the 10-20 nm range. The particle size could be readily controlled by capping and stabilizing agents. That is, increasing the concentration of the polymer, F-108, contributes to agglomeration in the media increasing the size of the particles, and a boost in the curcumin concentration led to a higher density of functional groups, resulting in smaller Au NPs. The Au NPs formed are found to be stable and crystalline. Gold nanoparticles present tremendous opportunities for the design of next-generation biosensors. The produced Au NPs could be successfully exploited to design an optical biosensor for glucose while acting as an immobilized encore. It is shown here that without exploiting the surface plasmon phenomena of metal nanoparticles, RRS spectroscopy can be useful to design a novel sensing scheme. It was demonstrated that the gold nanoparticles can detect glucose molecules with low energy consumption, fast response time, better selectivity and sensitivity with acceptable recovery. Mixing glucose and Au NPs further increased the RRS signal of the Au NPs. The proposed method offered a remarkable linear dynamic range, 0-10 mM. The proposed biosensor was stable both in the absence and presence of glucose. Serum and glucose samples were collected and tested as well to verify the efficiency of the method. Similar results were obtained with an excellent recovery for the serum sample verifying the performance of our method.

\section{Conflicts of interest}

There are no conflicts to declare.

\section{Acknowledgements}

Financial support provided by the Lebanese National Council of Scientific Research (NCSR), the Lebanon and American University of Beirut, Lebanon through URB as well as Kamal A. Shair Central Research Laboratory (KAS CRSL) facilities to carry out this work is greatly acknowledged.

\section{References}

1 C. Lopez-Cartes, T. C. Rojas, R. Litran, D. MartinezMartinez, J. M. de la Fuente, S. Penades and A. Fernandez, J. Phys. Chem. B, 2005, 109, 8761-8766. 
2 W. Chen, W. Cai, L. Zhang, G. Wang and L. Zhang, J. Colloid Interface Sci., 2001, 238(2), 291-295.

3 F. J. Heiligtag and M. Niederberger, Mater. Today, 2013, 16(7-8), 262-271.

4 A. L. Crumbliss, S. C. Perine, J. Stonehuerner, K. R. Tubergen, J. Zhao and R. W. Henkens, Biotechnol. Bioeng., 1992, 40(4), 483-490.

5 Y.-C. Yeh, B. Creran and V. M. Rotello, Nanoscale, 2012, 4, 1871-1880.

6 Y. Jin, P. Wang, D. Yin, J. Liu, L. Qin, N. Yu, G. Xie and B. Li, Colloids Surf., A, 2007, 302(1-3), 366-370.

7 S. C. Biradar and M. G. Kulkarni, RSC Adv., 2013, 3(13), 4261-4270.

8 M. Sengani, A. M. Grumezescu and V. D. Rejeswari, Open Nanomed., 2017, 2, 37-46.

9 A. L. González-mendoza and L. I. Cabrera-lara, Chem. Soc. Rev., 2015, 59(2), 119-129.

10 G. Sonavane, K. Tomoda and K. Makino, Colloids Surf., B, 2008, 66, 274-280.

11 C. L. Nehl, H. Liao and J. H. Hafner, Nano Lett., 2006, 6(4), 683-688.

12 T. K. Sau and C. J. Murphy, J. Am. Chem. Soc., 2004, 126(28), 8648-8649.

13 Y. Nagata, Y. Mizukoshi, K. Okitsu and Y. Maeda, Radiat. Res. Soc., 1996, 146, 333-338.

14 D. Radziuk, D. Grigoriev, W. Zhang, D. Su, H. Möhwald and D. Shchukin, J. Phys. Chem. C, 2010, 114(4), 1835-1843.

15 J. Song, D. Kim and D. Lee, Langmuir, 2011, 27, 13854-13860.

16 K. Ou, C. Yu, Y. Liu, K. Yang, C. Wang and Q. Chen, Mater. Res. Bull., 2011, 46(12), 2333-2337.

17 K. B. Narayanan and N. Sakthivel, Mater. Charact., 2010, 61(11), 1232-1238.

18 M. Shah, V. Badwaik, Y. Kherde, H. K. Waghwani, T. Modi, Z. P. Aguilar, H. Rodgers, W. Hamilton, T. Marutharaj, C. Webb, M. B. Lawrenz and R. Dakshinamurthy, Front. Biosci., 2014, 19, 1320-1344.

19 D. Patra, D. Ahmadieh and R. Aridi, Colloids Surf., B, 2013, 110, 296-304.

20 H. Hatcher, R. Planalp, J. Cho, F. M. Torti and S. V. Torti, Cell. Mol. Life Sci., 2008, 65, 1631-1652.

21 P. Pizzo, C. Scapin, M. Vitadello, C. Florean and L. Gorza, J. Cell. Mol. Med., 2010, 14(4), 970-981.

22 M. Stenvang, M. S. Dueholm, B. S. Vad, T. Seviour, G. Zeng, S. Geifman-Shochat, M. T. Sondergaard, G. Christiansen, R. L. Meyer, S. Kjelleberg, P. H. Nielsen and D. E. Otzen, J. Biol. Chem., 2016, 291(51), 26540-26553.

23 M. E. Egan, M. Pearson, S. A. Weiner, V. Rajendran, D. Rubin, J. Glockner-Pagel, S. Canny, K. Du, G. L. Lukacs and M. J. Caplan, Science, 2004, 304(5670), 600-602.

24 S. V. Jovanovic, S. Steenken, C. W. Boone and M. G. Simic, J. Am. Chem. Soc., 1999, 121(41), 9677-9681.

25 R. El Kurdi and D. Patra, Phys. Chem. Chem. Phys., 2017, 19(7), 5077-5090.

26 R. El Kurdi and D. Patra, Talanta, 2017, 168, 82-90.

27 D. Patra, D. Şen Karaman, D. Desai, E. El Khoury and J. M. Rosenholm, Mater. Res. Bull., 2016, 84, 267-272.
28 R. N. Moussawi and D. Patra, Sci. Rep., 2016, 6(1), 24565, DOI: $10.1038 /$ srep24565.

29 R. N. Moussawi and D. Patra, RSC Adv., 2016, 6(21), 17256-17268.

30 E. El Khoury, M. Abiad, M. Z. G. Kassaify and D. Patra, Colloids Surf., B., 2015, 127, 274-280.

31 A.-I. Moreno-Vega, T. Gomez-Quintero, R.-E. Nunez-Anita, L.-S. Acsota-Torres and V. Castano, Nanotechnology, 2012, 936041, DOI: 10.1155/2012/936041.

32 K. K. Jain, The Handbook of Nanomedecine, Springer, 2nd edn, 2008.

33 K. Cho, X. Wang, S. Nie, Z. Chen and D. M. Shin, Clin. Cancer Res., 2008, 14(5), 1310-1316.

34 K. Saha, S. Agasti, C. Kim, X. Li and V. Rotello, Chem. Rev., 2012, 112, 2739-2779.

35 D. J. Kim, S. M. Kang, B. Kong, W.-J. Kim, H.-j. H. Choi and I. S. Choi, Macromol. Chem. Phys., 2005, 206(19), 1941-1946.

36 B. J. Kim, J. Bang, C. J. Hawker and E. J. Kramer, Macromolecules, 2006, 39(12), 4108-4114.

37 L. Wang, N. N. Kariuki, M. Schadt, D. Mott, J. Luo, C.-J. Zhong, X. Shi, C. Zhang, W. Hao, S. Lu, N. Kim and J.-Q. Wang, Sensors, 2006, 6, 667-679.

38 Y. Kang and T. A. Taton, Angew. Chem., Int. Ed., 2005, 44(3), 409-412.

39 T. G. Drummond, M. G. Hill and J. K. Barton, Nat. Biotechnol., 2005, 21(10), 127-192.

40 J. Wang, Chem. Soc. Rev., 2008, 108, 814-825.

41 N. Chopra, V. Gavalas, L. G. Bachas and B. J. Hinds, Anal. Lett., 2007, 40(11), 2067-2096.

42 K. Kurihara and K. Suzuki, Anal. Chem., 2002, 74, 696-701.

43 P. Mergenthaler, U. Lindauer, G. A. Dienel and A. Meisel, Trends Neurosci., 2013, 36, 587-597.

44 S. F. Schakel, I. M. Buzzard and S. E. Gebhardt, J. Food Compos. Anal., 1997, 10(2), 102-114.

45 H. Goesmann and C. Feldmann, Nanoscience, 2010, 49(8), 1362-1395.

46 Y. Li, H. J. Schluesener and S. Xu, Gold Bull., 2010, 43(1), 29-41.

47 K. Sindhu, A. Rajaram, K. J. Sreeram and R. Rajaram, RSC Adv., 2014, 4(4), 1808-1811.

48 X. Huang and M. A. El-Sayed, J. Adv. Res., 2010, 1(1), 13-28.

49 M. Grzelczak, A. Sánchez-Iglesias, H. Heidari, S. Bals, I. Pastoriza-Santos, J. Perez-Juste and L. M. Liz-Marzan, ACS Omega, 2016, 1(2), 177-181.

50 J. Li, H. Feng, J. Li, Y. Feng, Y. Zhang, J. Jiang and D. Qian, Electrochim. Acta, 2015, 167, 226-236.

51 V. A. Marcolino, G. M. Zanin, L. R. Durrant, M. D. T. Benassi and G. Matioli, J. Agric. Food Chem., 2011, 59(7), 3348-3357.

52 J. Liu, J. Liu, W. Song, F. Wang and Y. Song, J. Mater. Chem. A, 2014, 2(41), 17477-17488.

53 A. P. Safronov, G. V. Kurlyandskaya, A. A. Chlenova, M. V. Kuznetsov, D. N. Bazhin, I. V. Beketov, M. B. Sanchez-Ilarduya and A. Martinez-Amesti, Langmuir, 2014, 30, 3243-3253. 
54 K. Sindhu, A. Rajaram, K. J. Sreeram and R. Rajaram, RSC Adv., 2014, 4(4), 1-11.

55 W. Zeng, H. Wang and Z. Li, J. Nanotechnol., 2016, 1-5.

56 E. Wijaya, C. Lenaerts, S. Maricot, J. Hastanin, S. Habraken, J.-P. Vilcot, R. Boukherroub and S. Szunerits, Curr. Opin. Solid State Mater. Sci., 2011, 15(5), 208-224.
57 E. Guerrero, M. A. Muñoz-Márquez, M. A. García, P. Crespo, E. Frenandez-Pinel, A. Hernando and A. Fernandez, Nanotechnology, 2008, 19, 175701.

58 K. Lacina, P. Skládal and T. D. James, Chem. Cent. J., 2014, 8, 1-17. 59 C. M. Wong, K. H. Wong and X. D. Chen, Appl. Microbiol. Biotechnol., 2008, 78(6), 927-938. 